\title{
Imaging of tumor infiltration of the pulmonary artery amenable to sleeve resection
}

Anna Maria Ciccone, MD, ${ }^{a}$ Antonio D'Andrilli, MD, ${ }^{a}$ Federico Venuta, MD, ${ }^{\text {b }}$ Claudio Andreetti, MD, and Erino A. Rendina, $M D^{\text {a }}{ }^{\text {Rome, Italy }}$

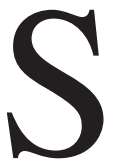

leeve resection of the pulmonary artery (PA) is rapidly gaining acceptance as an alterative to pneumonectomy in selected patients with locally advanced lung cancer. ${ }^{1-4}$ Contrary to bronchial sleeve resection, for which bronchoscopy is an important preoperative investigation tool, the evaluation of the PA is still uncertain. There is general agreement that computed tomography (CT) with the injection of contrast material is the most accurate technique, but it is often difficult, if not impossible, to discriminate between simple adhesion and infiltration of the vascular wall. ${ }^{4}$

\section{Clinical Summary}

Between 1989 and 2006, we performed 100 reconstructive procedures on the PA (sleeve, 36; patch, 55; conduit, 9), and all patients were preoperatively evaluated by CT. We report 3 emblematic cases showing possible discrepancies in terms of accuracy regarding vascular infiltration between preoperative evaluation by CT scan and intraoperative findings. It is noteworthy that the infiltration into the vascular lumen was clearly visible (Figure 1A, B) in

From the Division of Thoracic Surgery, University of Rome "La Sapienza," Sant'Andrea Hospital, ' Rome, Italy; and Division of Thoracic Surgery, University of Rome "La Sapienza," Policlinico Umberto I, ${ }^{\mathrm{b}}$ Rome, Italy.

Received for publication March 12, 2007; accepted for publication Aug 14, 2007.

Address for reprints: Anna Maria Ciccone, MD, Division of Thoracic Surgery, Sant'Andrea Hospital University of Rome "La Sapienza," Via di Grottarossa, 1035-39, 00189 Rome, Italy (E-mail: amciccone@ hotmail.com).

J Thorac Cardiovasc Surg 2008;136:229-30

$0022-5223 / \$ 34.00$

Copyright (C) 2008 by The American Association for Thoracic Surgery doi:10.1016/j.jtcvs.2007.08.030 only 1 case (case 1). This is the first fully documented case of sleeve resection of the PA reported in the literature. In this case, the tumor arising into the lumen of the PA did not infiltrate the origin of the lobar bronchus; thus, a bronchial sleeve resection was not required. The histologic diagnosis was squamous cell carcinoma. Conversely, CT can often be a source of misjudgment. Figure 2, $A$ shows a case of a left upper lobe tumor in which the infiltration of the PA is strongly suspected, but in which standard lobectomy was possible. In this case, because the tumor was close to the origin of the arterial branches to the upper lobe, clamping of the left PA at the origin allowed safe dissection so vascular reconstruction was not required. Figure 2, B, on the other hand, shows a case of a left upper lobe tumor in which segmental infiltration of the PA was indicated. On the basis of this CT study, PA sleeve resection was planned, but the tumor proved at operation to be unresectable. Extensive infiltration of the PA determined the unfeasibility of the vascular reconstruction at the operation. According to preoperative functional parameters, the risk associated with pneumonectomy was too high.

\section{Discussion}

Notwithstanding the evolution of conventional imaging (3-dimensional reconstructions) that has contributed to improve the accuracy of preoperative assessment, ${ }^{5}$ in a number of cases the presence of vascular involvement cannot be completely defined with preoperative imaging. It is difficult to plan a PA reconstructive procedure preoperatively, and the case in Figure 1 is unique. More often, the decision is made intraoperatively after accurate dissection of the involved structures. The control of the PA at its origin is crucial; often, clamping the PA allows the surgeon to plan and execute the resection safely, even if a sleeve is not necessary and the PA branches to the resected lobe can be tied conventionally. It

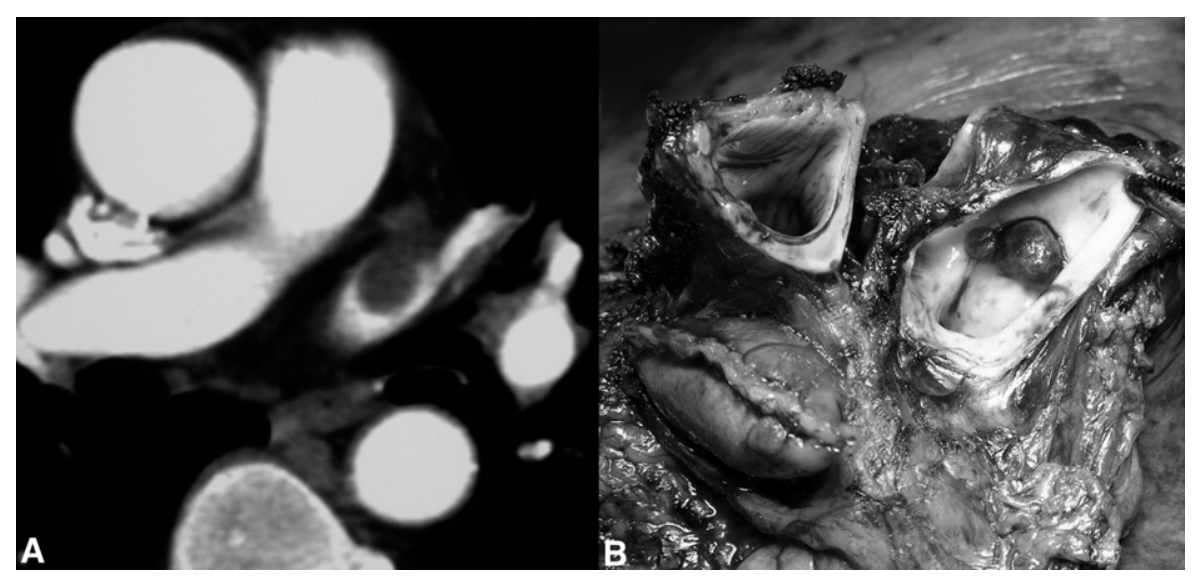

Figure 1. CT scan (A) and intraoperative view (B) of clearly visible tumor infiltration into the vascular lumen. 


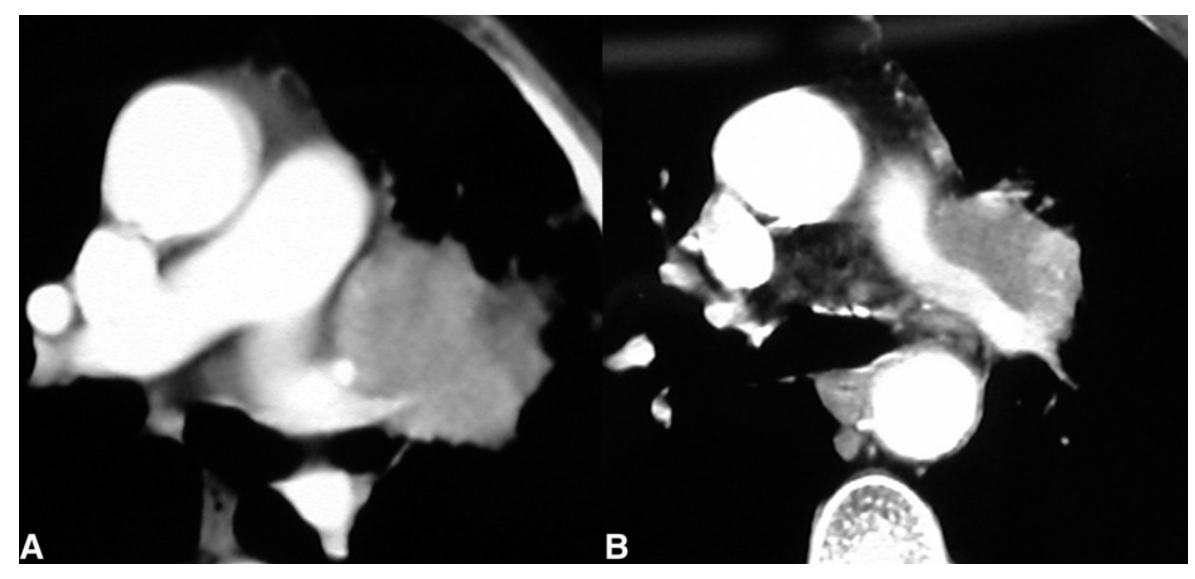

Figure 2. A, CT scan showing left upper lobe tumor with apparent infiltration into the PA that was not confirmed intraoperatively. B, CT scan showing a segmental infiltration of the PA by a left upper lobe tumor that was proved unresectable at the operation.

is important that the surgeon has familiarity with the interpretation of CT images and the vascular reconstructive techniques before approaching tumors involving the PA.

\section{References}

1. Vogt-Moykopf I, Fritz TH, Meyer G, et al. Bronchoplastic and angioplastic operation in bronchial carcinoma: long-term results of a retrospective analysis from 1973 to 1983. Int Surg. 1986;71:211-20.
2. Rendina EA, Venuta F, De Giacomo T, et al. Sleeve resection and prosthetic reconstruction of the pulmonary artery for lung cancer. Ann Thorac Surg. 1999;68:995-1002.

3. Yildizeli B, Fadel E, Mussot S, et al. Morbidity, mortality, and longterm survival after sleeve lobectomy for non-small cell lung cancer. Eur J Cardiothorac Surg. 2007;31:95-102.

4. Venuta F, Ciccone AM. Reconstruction of the pulmonary artery. Semin Thorac Cardiovasc Surg. 2006;18:104-8.

5. Hu Y, Malthaner RA. The feasibility of three-dimensional displays of the thorax for preoperative planning in the surgical treatment of lung cancer. Eur J Cardiothorac Surg. 2007;31:506-11. 